\title{
LA MUJER INDÍGENA CAMPESINA EN BOLIVIA Y SU OBJETIVO DE IGUALDAD. PROPUESTA DE APLICACIÓN DE UN MAPA ESTRATÉGICO BASADO EN VALORES COOPERATIVOS
}

\author{
POR \\ Fuensanta Carmen GALINDO REYES ${ }^{1}$ \\ José María DE LA VARGA SALTO² \\ Antonio Manuel CIRUELA LORENZO ${ }^{3}$
}

\section{RESUMEN}

Los condicionantes económicos y sociales presentes en el entorno rural de Bolivia han relegado a la mujer a un papel secundario y, en muchas ocasiones, injusto. El movimiento cooperativo y, concretamente su filosofía basada en una serie de valores, se presenta como una alternativa muy viable para cambiar la situación anteriormente comentada y conseguir que la mujer campesina indígena sea considerada en términos de igualdad en los contextos familiares y comunales. Sin embargo, necesitamos para ello una herramienta de aplicación, la cual ha consistido en una adaptación personal e innovadora del tradicional mapa estratégico propuesto por Kaplan y Norton (1992), a través del cual, la interrelación entre los objetivos correspondientes a diferentes perspectivas, contribuyen al alcance de la visión planteada.

Palabras clave: Entorno rural, Valores cooperativos, Mujer indígena campesina, Igualdad, Mapa estratégico

\footnotetext{
${ }^{1}$ Profesora Colaboradora. Departamento Economía y Administración de Empresas de la Universidad de Málaga. Dirección de correo electrónico: fcgr@uma.es

${ }^{2}$ Profesor Colaborador. Departamento Economía y Administración de Empresas de la Universidad de Málaga. Dirección de correo electrónico: jmdelavarga@uma.es

${ }^{3}$ Profesor Contratado Doctor. Departamento Economía y Administración de Empresas de la Universidad de Málaga. Dirección de correo electrónico: acl@uma.es

REVESCO N 107 - Primer Cuatrimestre 2012 - ISSN: 1885-8031 - www.ucm.es/info/revesco
}

DOI: 10.5209/rev_REVE.2012.v107.38742

Fecha de recepción: 25/01/2011

Fecha de aceptación: 23/03/2011 
Claves ECONLIT: J540, O180, P320, P490, Z100

\title{
THE INDIGENOUS RURAL WOMAN IN BOLIVIA AND THE EQUALITY AIM. A STRATEGIC MAP APPLICATION BASED ON COOPERATIVE VALUES
}

\begin{abstract}
The present economic and social conditions in rural areas of Bolivia have relegated women to a secondary role and, in many cases, unfair. The cooperative movement, and specifically its philosophy based on a set of values, is presented as a very viable alternative to change the situation discussed above and to make indigenous rural women to be considered in terms of equality in family and community contexts. However, we need to apply a tool, which has consisted of a personal and innovative adaptation of the traditional strategy map proposed by Kaplan and Norton (1992), through which the interrelationships between objectives from different perspectives, contribute the achievement of the outlined vision.
\end{abstract}

Keywords: Rural environment, Cooperative values, Indigenous rural women, Gender, Strategic map

\section{INTRODUCCIÓN: CONTEXTUALIZACIÓN Y JUSTIFICACIÓN}

América Latina atraviesa una etapa de gran dinamismo en relación a la consolidación democrática desde propuestas, escenarios y protagonismos marcados por la diversidad. Por primera vez, han accedido a la presidencia de algunos países latinoamericanos mujeres, como un signo inequívoco de cambio en estructuras fuertemente marcadas por la exclusión y la desigualdad.

Concretamente, en la región Andina el proceso de descentralización del estado surge en la década de los 80, con el fin de promover estados más eficientes, otorgando los gobiernos centrales mayores competencias y recursos a los gobiernos locales y comunidades (Mäckelmann, 2007). Aunque no todas las propuestas se materializaron en realidades, el enfoque de descentralización en Bolivia, modificó sustancialmente el marco económico-social dando paso a la presencia de nuevos protagonistas, como son los pueblos indígenas y las mujeres. 
En principio, el desarrollo de descentralización ha sido un proceso irregular y de controvertido éxito, siendo difícil encontrar ejemplos en los que el Estado haya delegado poderes a otros niveles sociales. Esta fragilidad hizo que, en sus inicios, este asunto no fuera especialmente atractivo para que las mujeres lo visualizaran como una oportunidad. Tanto en la concepción, como en el diseño de la descentralización, las mujeres y las comunidades indígenas han estado ausentes hasta fechas recientes (Díaz y otros, 2006).

Como resultado, los intereses y las preocupaciones de la mujer no están representados a los niveles de la formulación de políticas y la influencia de dicho colectivo es aún limitada en la toma de decisiones fundamentales en las esferas social, económica y política que afectan a la sociedad en su conjunto.

Precisamente ante esta realidad, Gallardo (2006) considera que uno de los desafíos para lograr la equidad de género consiste en garantizar la participación de las mujeres en estructuras de representación y de toma de decisiones. En este sentido, el ámbito comunal y productivo se presenta como un campo de posibilidades para dicha igualdad, pero también pleno de dificultades. Por eso, fortalecer los liderazgos para la participación de las mujeres en el ámbito de la comunidad y de la actividad productiva, se muestra como una medida improrrogable.

Los procesos de transformación política han dado lugar a la apertura de propuestas y espacios para incorporar e implementar actuaciones con el objetivo de lograr la equidad de género y el equilibrio entre el ámbito rural y el urbano. No obstante, abordar el concepto de género en sus diversas categorías, sociocultural, económica y política, es susceptible de transformación, ya que los roles de género se aprenden y dependen de las relaciones de variada naturaleza que los individuos establecen en una sociedad y en un momento histórico determinados.

América Latina tiene una historia particular y diferente a la de otros continentes. Bolivia en concreto, presenta una realidad característica, acentuada por su eminente heterogeneidad cultural y social y por un marcado proceso histórico y político del que han sido testigos. 
Bolivia es un país que cuenta con 9,8 millones de habitantes, 327 municipios, 36 grupos etnolingüísticos, que configuran una nación multilingüe y pluricultural de amplia diversidad de recursos naturales. El 50,1\% de su población es femenina, el $44 \%$ es menor de 18 años y el 60\% se identifica como indígena. Por otra parte, el $40 \%$ del total de la población y el 55\% de la población infantil viven en zonas rurales (INE de Bolivia, 2002).

En la actualidad, la mujer indígena campesina encara grandes dificultades en su vida cotidiana, en el interior de sus familias y comunidades, que impiden su pleno desarrollo social, obstaculizando el encuentro de la relación complementaria de su labor personal y productiva.

Según Bojanic y Otros (1994), el análisis de género ha mostrado que las mujeres cumplen roles específicos en el proceso de producción, en la gestión de la familia y de la comunidad, si bien, se ha revelado también un desigual acceso de éstas a los recursos productivos, a la información y a la toma de decisiones, especialmente en el ámbito rural.

La desigualdad en la participación de las mujeres en los diferentes aspectos tratados es una expresión, y en algunas circunstancias, efecto de las múltiples formas de inequidad de género que presenta la realidad social. Se trata de escenarios caracterizados por una distribución profundamente desequilibrada del peso de las adversidades entre los hombres y las mujeres (Montenegro, 2008).

El prejuicio de género, la desconsideración del ámbito rural y de la población nativa, provocan desigualdad de oportunidades básicas para la mujer indígena campesina, desde la falta de estímulos para desarrollar talentos personales, las dificultades en el acceso a una educación superior y a una preparación profesional, hasta la participación no equitativa en las funciones sociales de la familia y la comunidad.

Una vez establecido el marco en el que nos situamos, nos planteamos como objetivo del presente artículo, la propuesta de un camino que permita alcanzar un nivel de desarrollo personal, social y profesional de la mujer indígena campesina. En este sentido, consideramos muy adecuado establecer el cooperativismo, y más concretamente los principios y valores en los que éste se inspira, como un elemento fundamental para su consecución (Ciruela, 2008). 
Justificamos ahora, esta última afirmación. Las manifestaciones de ayuda mutua, de colaboración y de solidaridad se confunden con el propio desarrollo de la humanidad. La propia naturaleza del ser humano le lleva a no vivir aislado ya que, la asociación con otras personas, le permite hacer frente con mayor éxito a la satisfacción de sus necesidades personales, familiares y comunales.

En este sentido, el cooperativismo se presenta como una medida para hacer frente a situaciones de desigualdad, más si cabe, ante colectivos desfavorecidos, como en nuestro caso, la mujer indígena campesina en Bolivia. De hecho, estos tipos de sistemas de producción surgieron como nuevas fórmulas empresariales formadas por grupos sociales civiles en perjuicio o indefensión y que se basan, entre otros, en los valores de solidaridad y democracia (Barea y Monzón, 1992; Carrasco, 1993).

Estas organizaciones pretenden resolver problemas de grupos sociales en desventaja sin la ayuda directa de factores externos, es decir, son ellos mismos quienes lo consiguen mediante la unión y puesta en común de esfuerzo, sacrificio, trabajo y, en menor medida, capital. El eje básico de este tipo de iniciativas no es ni el dinero, ni los niveles de producción, ni la creación de empresas, sino la solidaridad. Solidaridad que, bien dirigida, permite generar empleo, riqueza colectiva y actividad económica en zonas donde no funcionan otras alternativas.

Para la consecución del objetivo anteriormente planteado, necesitamos establecer unas líneas de actuación, unas vías que en el largo plazo contribuyan al éxito de nuestra empresa, definiendo, en este caso la empresa como "la acción o tarea que entraña dificultad y cuya ejecución requiere decisión y esfuerzo" (RAE). Debemos definir una estrategia que conjugue los diferentes ámbitos de la situación analizada y que generen sinergias para la creación de valor, entendiendo como valor, la disminución de las desigualdades del colectivo desfavorecido al cual pretendemos ayudar, la mujer indígena campesina.

Para ello, presentamos el mapa estratégico como una herramienta que permite plasmar sintéticamente las vías para la consecución de valor. Dicho mapa es un modelo de sistemas que describe gráficamente la estrategia de una empresa a través de diferentes perspectivas, estableciendo objetivos específicos en cada una de ellas, así como las relaciones causa-efecto 
entre los mismos, buscando la complementariedad para el alcance del objetivo principal de este trabajo (Kaplan y Norton, 1996a).

El carácter integral de la herramienta y su simplicidad lo hace especialmente útil como un dispositivo de comunicación entre los diferentes grupos de interés involucrados en esta tarea.

\section{MARCO ESTRATÉGICO DE LA INVESTIGACIÓN}

Para establecer las vías que guían esta estrategia, es necesario definir previamente una serie de variables y realizar un diagnóstico para el análisis de la situación en que se encuentra. Debemos establecer, en este sentido, un marco estratégico que nos ayude a fijar los objetivos y nos oriente en las actuaciones a llevar a cabo posteriormente.

Dicho marco está integrado por la misión, la visión y los valores, así como por el análisis del entorno en el que se encuadra (Kaplan y Norton, 1996b), los cuáles se comentan en los siguientes epígrafes.

\subsection{Establecimiento de la misión}

La misión general de la empresa proporciona el punto de partida, definiendo por qué la empresa existe, es una declaración concisa de su razón de ser, el propósito básico hacia el que se dirigen sus actividades y las acciones que guían a sus actores. Atendiendo a esta definición, podemos concretar nuestra misión de la siguiente manera: "lograr la igualdad de la mujer indígena campesina boliviana en los diferentes ámbitos socio-económicos donde se integre".

\subsection{Establecimiento de la visión}

La visión presenta una imagen del futuro que aclara la dirección de la estrategia y ayuda a los actores implicados a comprender por qué y cómo deben apoyarla. Es una manifestación sucinta de los objetivos a medio y largo plazo. En nuestro caso, podemos establecer la siguiente visión: "que la mujer indígena campesina boliviana sea considerada en términos de igualdad en los contextos familiares y comunales". 


\subsection{Los valores cooperativos como alternativa de desarrollo de la mujer indígena campesina}

Los valores definen la forma en la que dirigimos la estrategia para alcanzar nuestra visión. Establecen el conjunto de principios, creencias y reglas que regulan la gestión de los objetivos. Constituyen el soporte del clima en el que se desarrollan. El establecimiento de valores permite contar con un marco de referencia que inspire y regule la forma de alcanzar nuestro objetivo general.

De forma más concreta, y considerando las características propias de las sociedades cooperativas, se deja entrever una base ideológica amplia y un fuerte arraigo a una serie de valores. Y así es, la Alianza Cooperativa Internacional (ACI) es la encargada de emitir y propugnar estos pilares básicos por los que se deben de guiar estas organizaciones, concretados en los denominados como principios y valores cooperativos (ACI, 1995).

Es justamente por la existencia de estos valores y principios por lo que hemos elegido al movimiento cooperativo como alternativa para el desarrollo y mejora de las condiciones de la mujer indígena campesina en Bolivia. Estos principios representan las guías de comportamiento y los objetivos específicos que establecen el funcionamiento y desarrollo de estas organizaciones. Pretenden conseguir una estructura eficaz y adecuada a los intereses de la sociedad en general y a los de sus socios en particular, permitiendo conjugar objetivos de naturaleza económica y social. $\mathrm{Y}$ es que, como se comentaba anteriormente, el cooperativismo se presenta como una medida para hacer frente a situaciones de desigualdad ante colectivos desfavorecidos.

Los principios no representan simples normas o criterios a aplicar, sino que son los fundamentos en los que se apoya la propia filosofía cooperativa (Sanchis, 1995), definiendo e impregnando su carácter. Su aceptación tácita e histórica los ha convertido en verdaderos baluartes del espíritu cooperativo y en el objetivo último y constante que deben mantener a lo largo de su existencia (García, 2000).

En cualquier caso, según la profesora Tous (1999), no es tan importante el cumplimiento exhaustivo de dichos principios, sino que al menos se siga su espíritu, y que la visión que cada principio proporciona esté arraigada en las actividades diarias. Esto permite 
que cualquier tipo de organización o grupo de personas, aunque no esté constituida como cooperativa, pueda desarrollar sus actividades basándose en dichos principios. Idea sobre la que gira nuestro trabajo y que da consistencia a la idea principal (incorporación de valores y principios cooperativos en las comunidades indígenas bolivianas).

Como se puede deducir de los párrafos anteriores, los principios cooperativos se inspiran en una serie de valores que son también concretados por la Alianza Cooperativa Internacional en, por un lado, "básicos globales" (autoayuda, responsabilidad, democracia, igualdad, equidad y solidaridad) y, por otro, "éticos" (honestidad, transparencia, responsabilidad y vocación social) (ACI, 1995).

De una manera resumida, el siguiente cuadro muestra los principios y valores inspiradores del comportamiento cooperativo.

Tabla 1. Principios y Valores Cooperativos

\begin{tabular}{|c|c|c|}
\hline $\begin{array}{c}\text { PRINCIPIOS } \\
\text { COOPERATIVOS }\end{array}$ & DEFINICION & $\begin{array}{c}\text { VALORES } \\
\text { COOPERATIVOS }\end{array}$ \\
\hline $\begin{array}{l}\text { Adhesión voluntaria y } \\
\text { abierta }\end{array}$ & $\begin{array}{l}\text { Las cooperativas son organizaciones voluntarias, } \\
\text { abiertas a todas las personas capaces de utilizar sus } \\
\text { servicios y dispuestas a aceptar las responsabilidades } \\
\text { de ser socio, sin discriminación social, política, } \\
\text { religiosa, racial o de sexo }\end{array}$ & Igualdad \\
\hline $\begin{array}{l}\text { Gestión democrática } \\
\text { por parte de los socios }\end{array}$ & $\begin{array}{l}\text { Las cooperativas son organizaciones gestionadas } \\
\text { democráticamente por los socios, los cuales } \\
\text { participan activamente en la fijación de sus políticas } \\
\text { y en la toma de decisiones. Los hombres y mujeres } \\
\text { elegidos para representar y gestionar las cooperativas } \\
\text { son responsables ante los socios }\end{array}$ & $\begin{array}{c}\text { Democracia e } \\
\text { igualdad }\end{array}$ \\
\hline $\begin{array}{c}\text { Participación } \\
\text { económica de los } \\
\text { socios }\end{array}$ & $\begin{array}{l}\text { Los socios contribuyen equitativamente al capital de } \\
\text { sus cooperativas y lo gestionan de forma } \\
\text { democrática. Al menos una parte de los activos es, } \\
\text { por regla general, propiedad común de la } \\
\text { cooperativa. Normalmente reciben una }\end{array}$ & Equidad \\
\hline
\end{tabular}




\begin{tabular}{|c|c|c|}
\hline & $\begin{array}{l}\text { compensación, si la hay, limitada sobre el capital } \\
\text { entregado como condición para ser socio. Los socios } \\
\text { asignan los excedentes para determinados fines: el } \\
\text { desarrollo de la cooperativa; la constitución de } \\
\text { reservas, de las cuales, al menos, una parte sería } \\
\text { irrepartible; el beneficio de los socios en proporción } \\
\text { a sus operaciones con la cooperativa; el apoyo de } \\
\text { otras actividades aprobadas por los socios }\end{array}$ & \\
\hline $\begin{array}{l}\text { Autonomía e } \\
\text { Independencia }\end{array}$ & $\begin{array}{l}\text { Las cooperativas son organizaciones autónomas de } \\
\text { autoayuda, gestionadas por sus socios. Si firman } \\
\text { acuerdos con otras organizaciones, incluidos los } \\
\text { gobiernos, o si consiguen capital de fuentes externas, } \\
\text { lo hacen en términos que aseguren el control } \\
\text { democrático por parte de sus socios y mantengan su } \\
\text { autonomía cooperativa }\end{array}$ & $\begin{array}{l}\text { Autoayuda, } \\
\text { democracia }\end{array}$ \\
\hline $\begin{array}{c}\text { Educación, formación } \\
\text { e información }\end{array}$ & $\begin{array}{l}\text { Las cooperativas proporcionan educación y } \\
\text { formación a los socios, a los representantes elegidos, } \\
\text { a los directivos y a los empleados para que puedan } \\
\text { contribuir de forma eficaz al desarrollo de sus } \\
\text { cooperativas. Informan al gran público, en especial a } \\
\text { los jóvenes y a los líderes de opinión, de la } \\
\text { naturaleza y beneficios de la cooperación }\end{array}$ & $\begin{array}{c}\text { Autorresponsabilidad, } \\
\text { honestidad }\end{array}$ \\
\hline $\begin{array}{c}\text { Cooperación entre } \\
\text { cooperativas }\end{array}$ & $\begin{array}{l}\text { Las cooperativas sirven a sus socios lo más } \\
\text { eficazmente posible y fortalecen el movimiento } \\
\text { cooperativo trabajando conjuntamente mediante } \\
\text { estructuras locales, nacionales, regionales e } \\
\text { internacionales }\end{array}$ & $\begin{array}{l}\text { Autoayuda, } \\
\text { solidaridad }\end{array}$ \\
\hline $\begin{array}{l}\text { Interés por la } \\
\text { comunidad }\end{array}$ & $\begin{array}{l}\text { Al mismo tiempo que se centran en las necesidades y } \\
\text { los deseos de los socios, las cooperativas trabajan } \\
\text { para conseguir el desarrollo sostenible de sus } \\
\text { comunidades, según los criterios aprobados por los } \\
\text { socios }\end{array}$ & Solidaridad \\
\hline
\end{tabular}

Fuente: Alianza Cooperativa Internacional, 1995

REVESCO No 107 - Primer Cuatrimestre 2012 - ISSN: 1885-8031 - www.ucm.es/info/revesco 
Centrándonos en el ámbito rural, en el cual se asienta el objeto de nuestro estudio (mujer indígena campesina), podemos comentar como, ante unos mercados cada vez más globalizados y competitivos, la cooperación se presenta como una de las fórmulas con las que cuenta el pequeño y mediano empresario agrario para mejorar su actividad y alcanzar ventajas en aspectos económicos, productivos, comerciales y, por supuesto, sociales.

La contribución de las sociedades cooperativas en los procesos de desarrollo territorial ha sido puesta de manifiesto por distintos autores (García-Gutiérrez, 1999; Coque, 1999; Bel, Buendía y Rodrigo, 2000; Mozas y Rodríguez, 2000; Juliá y Marí, 2002; Mozas y Bernal, 2006; Juliá, García y Meliá, 2006), así como por la propia Unión Europea, que destaca las posibilidades, no sólo de mejoras empresariales o económicas (empleo, infraestructuras...), sino también la integración social y humana en estos entornos (DOCE 11/02/94), y siempre desde un punto de vista endógeno que frena la salida de capitales y recursos a otras zonas (DOCE 14/09/1987).

Más concretamente, la Comisión Europea (Conferencia Europea de Salamanca, 2002) reconoció que las formas jurídicas integrantes de la Economía Social son, para determinados sectores, una alternativa muy viable en el sostenimiento del estado de bienestar, ya que permiten la generación de empleo y fomentan la capacidad emprendedora.

En definitiva, para alcanzar dicho desarrollo la empresa se convierte en un eslabón que conecta la sociedad y la economía. Y para trasladar las necesidades y objetivos de la comunidad a la empresa, se propone que la estructura societaria responda al concepto de cooperativa, considerándola como la más adecuada para que los resultados económicos tengan el carácter de endógenos y sostenibles (Cabaleiro y Fernández-Feijóo, 2007).

Y esto es así, en buena parte, porque las metas y objetivos que argumentan y dan consistencia al desarrollo rural coinciden, o son perfectamente compatibles, con las ideas fundamentales del movimiento cooperativo. De esta forma, el concepto de desarrollo rural persigue tres objetivos básicos (Rodríguez, 2002): a) alcanzar y mantener estructuras económicas y empresariales eficientes; b) lograr la equidad social compatible con la eficiencia económica; c) garantizar el desarrollo duradero del territorio. 
Objetivos que enlazan con la forma de actuación y postulados del cooperativismo, así como con el objeto establecido en este artículo, esto es, el desarrollo personal y profesional de la mujer indígena campesina mediante la puesta en común de recursos, esfuerzo y conocimientos, así como una participación de los integrantes en la estructura orgánica y operacional de las comunidades y una toma de decisiones con base democrática.

Pero sin duda es uno de los principios cooperativos (ACI, 1995) el que deja una más clara evidencia de la relación tan estrecha existente entre el cooperativismo y el territorio físico y social donde se desarrolla la actividad. Nos referimos al principio de "interés por la comunidad", el cual establece, como antes se comentaba, que las cooperativas, al mismo tiempo que se centran en las necesidades y deseos de sus integrantes, trabajan para conseguir el desarrollo sostenible de sus comunidades. En definitiva, coloca a las sociedades cooperativas en el centro de los procesos de desarrollo local (urbano y rural) desde una triple perspectiva: económico, social y ambiental (Buendía, 1999).

Principio que unido a otros como el de "inter-cooperación, autonomía o formación" (ACI, 1995), erigen a las cooperativas como precursoras de un proceso de transformación y generación económica de los espacios rurales, abogando por una actividad socialmente útil, capaz de articular y defender las necesidades de los sectores más débiles, que incorpore valores éticos y sitúe a la persona, y en nuestro caso a la mujer indígena campesina, en el centro del proyecto social y económico (García, 2000).

\subsection{Descripción de los grupos de interés}

Con el término "grupo de interés", y siguiendo la metodología del mapa estratégico de Kaplan y Norton (1992), nos referimos a los colectivos, ya sean individuales o grupales, que afectan al objeto de nuestro estudio (mujer indígena campesina) y que, por tanto, experimentarán algún tipo de cambio en el desarrollo de nuestra misión. Dichos grupos de interés pueden ser contextualizados de la siguiente forma, en base al análisis efectuado durante nuestra visita a la zona:

- Familia: nos encontramos con una estructura familiar totalmente patriarcal, donde el hombre, en muchas ocasiones, toma las decisiones y la mujer las ejecuta. En este sentido, la mujer se encarga tanto de la parte reproductiva (cuidado y mantenimiento 
del hogar y sus integrantes) como de la parte productiva (actividades agrarias y/o agropecuarias), sufriendo, en muchos casos, situaciones de injusticia, incomprensión e incluso malos tratos físicos y psíquicos.

- Comunidad: también con estructura patriarcal, las normas y cultura en general de muchas comunidades es contraria totalmente al desarrollo personal y profesional de la mujer. Son hombres los que toman las decisiones y los que poseen la propiedad de las tierras, lo cual dificulta enormemente el debido protagonismo femenino.

- Nación: desde la llegada de la nueva mentalidad política a Bolivia, son numerosas las actividades en defensa del hombre y mujer indígenas y, especialmente, los afincados en zonas rurales. Sin embargo, aún no son visibles los verdaderos efectos de dichas medidas, al menos en lo que a gestión política se refiere.

- Internacional: son diversos los proyectos de cooperación internacional al desarrollo que se han puesto en marcha en Bolivia para conseguir una verdadera igualdad de género. En el caso concreto de España, la AECID (Agencia Española de Cooperación Internacional para el desarrollo) mantiene una línea abierta sobre dicha temática.

En términos generales, la posible consecución de los objetivos que vamos a establecer en el presente artículo, va a suponer importantes repercusiones para los distintos grupos de interés, especialmente para la familia y la comunidad, donde el hombre, necesariamente, va a tener que concienciarse de dicha igualdad y cambiar, en muchas ocasiones, hábitos sociales y personales muy arraigados en su cultura, pero que van en contra de una filosofía de equidad y progreso de la mujer.

\subsection{Análisis del entorno}

Además de los aspectos comentados en los epígrafes anteriores, se han realizado análisis PEST y DAFO que permiten una visión real y cercana de las circunstancias y características del colectivo estudiado, desde las perspectivas política, económica y social. 


\subsubsection{Análisis PEST}

La herramienta utilizada en el presente artículo (mapa estratégico) requiere de una previa contextualización sobre el objeto de estudio. Para ello se ha realizado un análisis PEST del entorno general boliviano, donde se ponen de manifiesto algunas de las circunstancias más significativas a nivel Político-Legal, Económico, Socio-Cultural y Tecnológico, y que, de alguna manera influyen en la situación y posibilidades de desarrollo de la mujer indígena campesina boliviana. La tabla 2 muestra dicho análisis, comentando algunos de dichos aspectos posteriormente.

Tabla 2. Análisis PEST

\begin{tabular}{|c|c|}
\hline POLITICO-LEGAL & ECONOMICO \\
\hline $\begin{array}{l}\text { - Relativa estabilidad gubernamental en } \\
\text { ámbito nacional, regional y municipal } \\
\text { - Objetivos programa político partido } \\
\text { MAS (favorecedor mujer, } \\
\text { campesinado, indígena...) (1) } \\
\text { - Programa de apoyo al desarrollo de } \\
\text { otros países } \\
\text { - Incorporación a la Alianza } \\
\text { Bolivariana para los Pueblos de } \\
\text { Nuestra Tierra (ALBA) } \\
\text { Nueva estrategia de desarrollo rural } \\
\text { sostenible (2) } \\
\text { Avances en Política Internacional }\end{array}$ & $\begin{array}{l}\text { - Incremento de la economía informal } \\
\text { - } \text { (del } 60 \text { al } 70 \% \text { en relación al PIB) } \\
\text { - } \text { Población bajo la línea de pobreza } \\
\text { (64\%), más acrecentada en el ámbito } \\
\text { rural (82\%) (6) } \\
\text { - Pobreza extrema (36\% población total } \\
\text { y } 56 \% \text { de la rural) } \\
\text { - Coeficiente de Gini (0,6). } 2^{\circ} \text { país más } \\
\text { desigual, tras Brasil } \\
\text { - Propuesta nacionalización } \\
\text { hidrocarburos } \\
\text { - Fomento del sector minero } \\
\text { Creación del Banco de Desarrollo } \\
\text { Productivo (BDP) (7) } \\
\text { Mejora situación económica general }\end{array}$ \\
\hline SOCIO-CULTURAL & TECNOLÓGICO \\
\hline $\begin{array}{l}\text { - Migración de hombres hacia ciudades } \\
\text { - Amplia emigración internacional }\end{array}$ & $\begin{array}{l}\text { - Desigualdad general entre medio rural } \\
\text { y urbano }\end{array}$ \\
\hline
\end{tabular}

REVESCO No 107 - Primer Cuatrimestre 2012 - ISSN: 1885-8031 - www.ucm.es/info/revesco 
- Papel secundario de la mujer (3)

- Problema de acceso a agua potable (8)

- Reforma educativa (4)

- Problemas de saneamiento básico

- Fragmentación social, regional y - Problemas de electrificación étnica

- Dificultades sanitarias severas (9)

- Tradicional descrédito de las - Fomento de nuevas alternativas de instituciones democráticas

- Renta Dignidad (5) transporte

- Acceso a las telecomunicaciones (10)

Fuente: Elaboración propia a partir de Ciruela y Otros, 2011

(1) El Programa de Gobierno del Movimiento al Socialismo (2010-15) recoge una serie de propuestas y sugerencias hechas por los diversos sectores de la sociedad, las cuales apuntan a un nuevo objetivo común: avanzar en el proceso de cambio para llevar a Bolivia a ser un país líder. Para ello, según establece el mismo partido, se necesita el trabajo de todos, por lo que se lanza una invitación a la ciudadanía para que participe en la construcción de un país con justicia, igualdad y dignidad.

(2) La nueva estrategia de desarrollo rural supone un hito en la historia de Bolivia. Se considera que el sector agropecuario debe seguir consolidando el desarrollo productivo rural, de manera integral y sustentable, fomentando los emprendimientos económicos comunitarios, así como la transformación y comercialización de la producción agropecuaria convencional y ecológica que genere excedentes, ingresos y empleos.

Además, se piensa promover la gestión sostenible de los recursos naturales y de la biodiversidad, la preservación, conservación, defensa y mejora del medio ambiente, garantizando el derecho a la tenencia y acceso a la tierra, eliminando definitivamente el latifundio y consolidando el derecho al uso del agua para riego, así como la revalorización de la hoja de coca con respeto a los saberes locales, usos y costumbres de las naciones y pueblos indígenas originarios.

(3) Una de las principales preocupaciones del Gobierno actual ha sido la erradicación de la pobreza que, en gran medida, afecta a las áreas indígenas rurales y, en especial, a la mujer 
campesina. Para ello se ha creado un determinado Bono económico que beneficia a todas las mujeres embarazadas y a los niños menores de 2 años (denominado Juancito Pinto) (Diario el Día)

(4) En 2007, se crean las bases teórico-prácticas y se establecen las normas del nuevo modelo educativo de carácter socio comunitario productivo, y que postula los siguientes principios (Ministerio Educación Bolivia):

- Educación descolonizadora, liberadora, revolucionaria y transformadora.

- Educación comunitaria, democrática, participativa y de consensos.

- Educación intracultural, intercultural y plurilingüe.

- Educación productiva y territorial.

- Educación científica, técnica, tecnológica y artística.

(5) Mediante la Ley 3791 del 28 de noviembre de 2007, se establece el pago de la Renta Universal de Vejez, conocida como Renta Dignidad, que forma parte del Régimen de Seguridad Social no contributivo. La Renta Dignidad es una prestación vitalicia otorgada a todos los bolivianos de 60 años o más que residen en el país.

(6) La incidencia de pobreza extrema o indigencia mide el porcentaje de personas que vive con un ingreso inferior a la canasta básica alimentaria. Del año 1996 al 2008, el porcentaje de personas en dicha situación se ha reducido en 9,4 puntos porcentuales (del 41,2\% en 1996 al $31.8 \%$ en 2008). Si se analiza este aspecto por ámbitos, se observa que en la actualidad, la pobreza extrema ha disminuido tanto en el urbano como en el rural, siendo especialmente significativa la reducción en éste último (del 62,9\% en 2005 al 49,2\% en 2008). (UDAPE Bolivia)

(7) En el año 2007 se creó el Banco de Desarrollo Productivo (BDP), a través del cual se canalizan recursos de largo plazo en condiciones ventajosas (interés del 6\%, plazos de hasta 12 años y periodos de carencia variables), que tienen como finalidad reestructurar el sistema productivo, dirigiendo principalmente los créditos a emprendedores, micro y pequeñas unidades, así como a asociaciones de productores. 
(8) Hasta finales de 2005, aproximadamente 2,3 millones de habitantes no tenían acceso al agua potable, de los cuales un $75 \%$ correspondía al área rural. Por otra parte, cerca de cinco millones de personas no tenían acceso a un adecuado servicio de saneamiento; de éstos, un 57\% también se asentaba en dicha área (UDAPE Bolivia)

(9) La situación de la salud se puede resumir en la existencia de una marcada concentración y duplicidad de la oferta de servicios en los centros urbanos y, por el contrario, muy poca o ninguna oferta en las áreas rurales. A esto hay que añadir la insuficiencia de atención en los servicios del sistema de salud, la falta de aplicación de criterios de calidad y eficiencia, y por supuesto, la carencia en infraestructura, equipamiento y personal adecuado.

(10) Con la promulgación del Decreto Supremo del 1 de mayo de 2008 para la Nacionalización de la Empresa Nacional de Telecomunicaciones (ENTEL) el servicio de telecomunicaciones se convierte en un derecho fundamental para todos los bolivianos, tal y como se establece en la Nueva Constitución Política del Estado.

\subsubsection{Análisis DAFO}

El análisis DAFO, como síntesis de las características socio-económicas de la mujer indígena campesina boliviana, permite determinar un diagnóstico adecuado de la situación, así como orientar y justificar los objetivos y ámbitos de intervención correspondientes a las diferentes perspectivas estratégicas de nuestro estudio. Buena parte de las mismas van encaminadas a potenciar las fortalezas y reducir las debilidades, y para ello, hay que también aprovechar las oportunidades y superar las amenazas que se presentan en el ámbito de actuación que nos ocupa. 
Tabla 3. Análisis DAFO

\begin{tabular}{|c|c|}
\hline $\begin{array}{c}\text { ASPECTOS A MINORAR } \\
\text { (DEBILIDADES Y AMENAZAS) }\end{array}$ & $\begin{array}{c}\text { ASPECTOS A POTENCIAR } \\
\text { (FORTALEZAS Y } \\
\text { OPORTUNIDADES) }\end{array}$ \\
\hline $\begin{array}{l}\text { - } \text { Arraigado factor cultural } \\
\text { - } \text { Escaso nivel de formación } \\
\text { - } \text { Falta de profesionalización } \\
\text { - } \text { Dificultad de acceso de la mujer a } \\
\text { - } \text { Nosestructuración familiar } \\
\text { - } \text { Asociacionismo débil } \\
\text { - } \text { Discriminación de género } \\
\text { - } \text { Falta de acceso a la información } \\
\text { - Falta de formación e información en } \\
\text { perspectiva de género }\end{array}$ & $\begin{array}{l}\text { - Mayor presencia en juntas vecinales y } \\
\text { asociaciones locales } \\
\text { - Estructura de población joven y } \\
\text { crecimiento vegetativo importante } \\
\text { - Diversidad interna, elevada riqueza } \\
\text { paisajística e importante significación } \\
\text { ambiental } \\
\text { - Reformas legales en defensa de los } \\
\text { derechos de la mujer } \\
\text { - Vocación de servicio y liderazgo del } \\
\text { gobierno de las comunidades } \\
\text { - Fomento y desarrollo de los } \\
\text { microcréditos de } \\
\text { Incremento de programas de } \\
\text { cooperación internacional }\end{array}$ \\
\hline
\end{tabular}

Fuente: Elaboración propia a partir de Ciruela y Otros (2011) y UDAPE Bolivia

El presente análisis DAFO (tabla 3) nos muestra en términos generales, determinados aspectos que reflejan la problemática de la mujer indígena campesina en Bolivia. Circunstancias. como la escasez de formación y profesionalización, la falta de acceso a la información, la discriminación de genero, la sobrecarga de trabajo y responsabilidades, la inexistencia de instancias de apoyo y protección a las mujeres o la debilidad en aspectos estructurales, que vislumbran y potencian el papel secundario de la mujer indígena campesina boliviana, y que volverán a ser objeto de análisis en el epígrafe 3.2., con motivo de justificar el establecimiento de los objetivos planteados en cada perspectiva. 


\section{CONSTRUCCIÓN DEL MAPA ESTRATÉGICO}

Durante este epígrafe, siguiendo como comentábamos, la metodología propuesta por Kaplan y Norton (1992, 1993 y 1996), planteamos los principales pasos, que nos han permitido la construcción final del mapa estratégico objeto de nuestra investigación, tal y cómo se recoge en la figura 2 de este trabajo.

En este sentido, consideramos que un elemento fundamental del citado mapa, es la determinación de los procesos por los que se crea valor, en base a una serie de intangibles de la "empresa" que permiten llegar a la visión, en nuestro caso, "que la mujer campesina indígena boliviana sea considerada en términos de igualdad en los contextos familiares y comunales". Pues bien, nuestra propuesta de valor se basa en la aplicación de los principios y valores cooperativos para la consecución de la visión planteada.

\subsection{Perspectivas para la creación de valor}

La consecución de valor supone en nuestro caso la concatenación de una serie de perspectivas de actuación, las cuales pueden definirse a priori mediante las siguientes preguntas:

Perspectiva Personal: ¿Cuáles son las expectativas que tiene la mujer campesina indígena para la consecución de nuestra visión?

Perspectiva Familiar: Para alcanzar nuestra visión, ¿qué cambios se deberían producir en el ámbito familiar?

Perspectiva Comunal: ¿Cómo mejorar las relaciones económico-sociales de la comunidad a través de la incorporación de los principios y valores cooperativos?

Perspectiva Nacional: ¿Qué aspectos se deben fomentar desde el ámbito nacional para apoyar el proceso de igualdad de la mujer campesina indígena?

Perspectiva Internacional: ¿Cómo puede contribuir el entorno internacional para la consecución de la visión planteada y en el proceso de creación de valor? 
Estas perspectivas, y más concretamente los cambios acontecidos en las mismas permitirán alcanzar la visión establecida (figura 1).

Figura 1. Relación de las perspectivas y la visión planteada

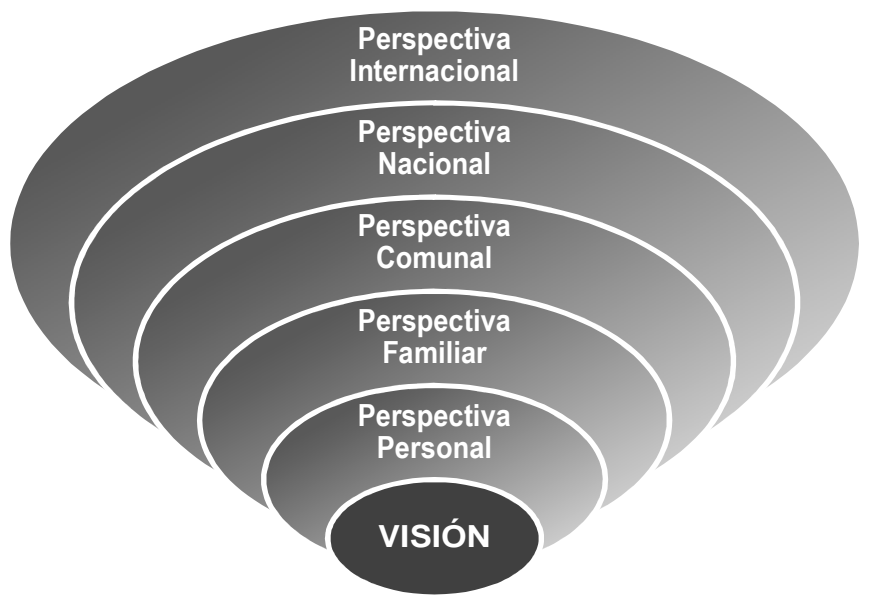

Fuente: Elaboración propia

\subsection{Objetivos estratégicos por perspectivas}

A continuación se describen los objetivos planteados en cada perspectiva, para cuya concreción han sido tenidos en cuenta las circunstancias y características obtenidas anteriormente en los análisis PEST y DAFO.

\section{a) Perspectiva Personal:}

La discriminación de género en la familia, en las comunidades rurales y en la vida diaria lleva a la mujer a estar más expuesta al maltrato físico y psicológico por parte del varón, así como a la sobrecarga de trabajo y de responsabilidades, lo cual está íntimamente relacionado con una falta de motivación, baja autoestima y ausencia de confianza en sí misma. Situación que se ve agravada, en muchas ocasiones, por la inexistencia de instancias de apoyo y protección a las mujeres. 
De esta forma, el objetivo planteado en esta perspectiva queda definido como la realización personal y profesional de la mujer indígena campesina. Esto supondrá, entre otras cosas, una mayor motivación, valoración y autoconfianza.

\section{b) Perspectiva Familiar:}

Los objetivos planteados en esta perspectiva son los siguientes:

- Incrementar el bienestar. Existen una serie de aspectos estructurales y coyunturales, como pueden ser, por ejemplo, déficits en infraestructuras de viviendas o escasez de medios sanitarios, que dificultan el quehacer diario de la mujer en sus ámbitos familiar y productivo. Ante ello, este objetivo plantea el logro de mejoras en el contexto del hogar, como pueden ser higiénico-sanitarias, alimenticias, servicios básicos...

- Educar en igualdad. La falta de formación con perspectiva de género desde la infancia, sigue siendo una asignatura pendiente tanto para el colectivo masculino como para el femenino, y está presente tanto en el ámbito escolar como en el familiar. A ello le unimos la escasez de acciones educativas sobre derechos sexuales y reproductivos.

Por tanto, resulta fundamental cambiar, en todos los miembros de la familia (hombres, niños e incluso las propias mujeres), la concepción de los roles tradicionales ejercidos por la mujer.

- Consolidar el núcleo familiar. La creciente concentración de la población en áreas urbanas en detrimento del medio rural conlleva a un estancamiento y reducción de la población en los pequeños municipios, lo que ahonda los desequilibrios intraterritoriales. En concreto, la migración del varón por pobreza rural hacia las ciudades unida a la emigración internacional obliga a muchas mujeres a incrementar el número de responsabilidades $\mathrm{y}$, por tanto, a reducir su tiempo disponible para otras actividades personales. En cuanto a la migración de la mujer, también ha causado efectos importantes en el interior de las familias, a causa de la desintegración de las mismas y también en los hijos que han quedado sin el apoyo afectivo, la seguridad y orientación de sus padres. 
Ante esta situación se propone el siguiente objetivo: mantener la estructura equilibrada que aportan el hombre y la mujer dentro de la unidad familiar, la cual se está debilitando por aspectos como la emigración, el traslado del hombre a la ciudad...

\section{c) Perspectiva Comunal:}

El objetivo principal de esta perspectiva es introducir y fomentar los principios y valores cooperativos, a través de los cuales, pretendemos alcanzar otra serie de objetivos relacionados con la comunidad. La descripción de los mismos y la relación de éstos con los principios cooperativos se establecen a continuación:

- Incrementar asociacionismo. En determinadas zonas, no existe un movimiento asociativo suficiente por parte de la mujer a nivel general, por lo cual, le resulta más difícil conocer y defender sus derechos. Por ello establecemos como objetivo que la mujer se involucre más en las asociaciones existentes, así como que se creen nuevas organizaciones. Esto se podría alcanzar con la puesta en práctica de los principios de adhesión voluntaria y abierta y de cooperación entre cooperativas, los cuáles permitirían un acceso con menos restricciones de la mujer a dichas asociaciones, así como la creación, a través de redes, de instituciones supracomunitarias con las que se podrían alcanzar sinergias de tipo social y económico.

- Participación real en la toma de decisiones. La falta de profesionalización derivada, entre otras causas, por la escasa formación, dificulta el acceso de la mujer a cargos de poder en los ámbitos público y privado y, por tanto, su participación en los momentos de deliberación, formulación de políticas públicas y toma de decisiones, quedando su papel relegado a tareas propias del hogar y del ámbito productivo básico. Por tanto se establece este principio como la necesidad de que la mujer participe de manera activa, sin relegar su papel a una presencia simbólica, teniendo voz y voto en aquellas decisiones relevantes para la comunidad. Esto se podría fomentar mediante la puesta en práctica del principio gestión democrática por parte de los socios, el cual establece iguales derechos de voto y toma de decisiones colectivas.

- Dar valor a la diversidad cultural. Teniendo en cuenta las diferencias culturales entre las distintas etnias existentes en Bolivia, consideramos que el principio de autonomía $e$ independencia, podría contribuir a poner de relevancia los contrastes entre cada comunidad, destacando sus aspectos más interesantes, con objeto de establecer actividades económicas 
generadoras de valor añadido en dichas comunidades, basadas, por ejemplo, en el turismo o la comercialización artesanal.

- Fomentar el grado de implicación. Nos referimos con este objetivo a cambiar la estructura organizativa basada en el trabajo individual de cada familia por actividades de carácter conjunto y compartido, tanto desde un punto de vista social como económico. Para ello, consideramos conveniente, tal y como establece el principio participación económica de los socios, que cada familia se implique, no sólo desde un punto de vista personal sino también laboral y económico (por ejemplo, mediante la aportación de cuotas en función de su capacidad económica o productiva).

- Mejorar los sistemas de producción y comercialización. Consideramos muy importante para las mejoras personales y sociales planteadas en la visión, el desarrollo eficaz y eficiente de las actividades agrarias, ganaderas y artesanales, tanto en sus facetas productivas como comercializadoras, las cuáles servirán como motor económico e impulso social y comunitario. Ello se vería facilitado por la implantación del principio participación económica de los socios, ya que supondría un esfuerzo e implicación conjunto de parte de la Comunidad.

Además, el entorno rural boliviano presenta una gran diversidad, una elevada riqueza paisajística y una importante significación ambiental, lo cual propicia, como antes comentamos, que el turismo rural se presente como una oportunidad de diversificación de la actividad y de las rentas agrarias.

- Mejorar las infraestructuras. La puesta en práctica del principio de interés por la comunidad, el cual establece que, al mismo tiempo que se satisfacen las necesidades de los socios, también es necesario velar por los intereses del entorno en el que se ubican las comunidades, conllevaría mejoras significativas y sostenibles en aspectos tales como higiénico-sanitarios, infraestructuras básicas,...

Por poner un ejemplo de dichas necesidades, y aunque la situación ha mejorado en los últimos años, el acceso a electricidad en el ámbito rural es todavía muy limitado; solo un $45 \%$ de los hogares situados en el área rural tienen acceso, frente al 90\% en el área urbana. 
- Fomentar la capacitación. En materia de educación, existen en la actualidad en el ámbito rural una serie de circunstancias adversas, como pueden ser los elevados índices de analfabetismo o el abandono precoz del sistema educativo, que afectan especialmente a la mujer (el $40 \%$ no ha recibido ningún tipo de instrucción). Esta situación, entre otras causas, provoca una elevada incidencia en el desempleo de dicho colectivo, que es junto a los jóvenes, el que presenta una mayor dificultad para acceder al mercado de trabajo.

Por ello consideramos fundamental la capacitación de las mujeres para alcanzar sus metas personales y profesionales. En este sentido, el principio de educación, formación e información, puede suponer un apoyo fundamental hacia la búsqueda de las verdaderas necesidades formativas y la satisfacción de éstas.

Este mismo principio, en términos generales, ayudaría a dar a conocer en la comunidad la naturaleza y beneficios de la cooperación, actividad que consideramos se debería llevar a cabo de manera prioritaria, como forma de concienciación colectiva.

\section{d) Perspectiva Nacional:}

- Fomentar las políticas educativas. Resulta fundamental el apoyo por parte de las instituciones gubernamentales de iniciativas dirigidas hacia la disminución de diferencias en cuanto a los niveles educativos de la población. Aunque fomentando, al mismo tiempo, la idiosincrasia cultural propia de cada grupo étnico.

- Fomentar la educación a nivel de género. Es necesaria la educación en igualdad tanto en el ámbito escolar como familiar, siendo las nuevas generaciones las encargadas de cambiar la concepción actual de la mujer.

- Fomentar las políticas de conciliación. Los distintos roles ejercidos por la mujer en su vida cotidiana y la necesidad de su compatibilización real, hacen convenientes la puesta en marcha de mecanismos, a nivel nacional, que permitan conciliar su vida familiar y profesional.

- Incrementar políticas de financiación. El desarrollo del componente social y personal de la mujer requiere la concesión de apoyo económico a nivel nacional, el cual se podría complementar con los recursos obtenidos a nivel endógeno por la propia comunidad. 


\section{e) Perspectiva Internacional:}

- Mantener e incrementar políticas de ayudas sociales y económicas. Resulta fundamental seguir contando con el apoyo internacional, tanto en los aspectos sociales como económicos, ya que no podemos olvidar que Bolivia es uno de los países con mayor índice de pobreza en Latinoamérica.

En este sentido comentar que los programas que actualmente se desarrollan giran en torno a las siguientes temáticas: fortalecimiento de la sociedad civil, desarrollo agrario alternativo, mujer y desarrollo o desarrollo rural (AECID).

\subsection{Relaciones causa-efecto entre objetivos estratégicos}

A continuación se explican las relaciones causa-efecto existentes entre los objetivos estratégicos anteriormente descritos, las cuales van a permitir alcanzar la visión propuesta.

El apoyo internacional constituye el soporte elemental para la consecución de los objetivos formulados en las distintas perspectivas. Cómo antes apuntábamos, Bolivia es uno de los países más pobres a nivel mundial, y sin este sustento, hubiese sido mucho más difícil alcanzar los niveles de desarrollo social y económico de los últimos años.

Al mismo tiempo, consideramos conveniente establecer políticas y acciones conjuntas por parte del gobierno nacional, de forma que se alcancen sinergias y una mayor eficiencia en el uso y empleo de los fondos nacionales e internacionales. Dichos fondos son destinados, entre otras causas, a la financiación de actividades educativas, la concienciación de género o el establecimiento de actividades empresariales, los cuáles, como antes comentamos, pueden significar un apoyo vital en pro de la visión planteada en este artículo.

La gran diversidad cultural y étnica existente en Bolivia nos plantea la necesidad de focalizar nuestra estrategia en las distintas comunidades que conforman el panorama nacional. Además, dichas comunidades, presentes en el ámbito rural y de origen indígena, son las que cuentan con una mayor problemática económica y social, como ya se ha puesto de referencia en los análisis DAFO y PEST anteriormente realizados. Ello ha obligado al gobierno a impulsar medidas de avance de dichos colectivos, aunque consideramos que en determinadas 
ocasiones no han sido efectivas ni suficientes, por lo que continúan existiendo, fundamentalmente en el ámbito rural, una serie de necesidades insatisfechas. Para ello, es decir, para cubrir tales deficiencias, planteamos la incorporación de la filosofía cooperativa en el seno de las colectividades indígenas campesinas, centrándonos fundamentalmente en la mejora de las condiciones personales y sociales de la mujer, como colectivo especialmente desfavorecido.

Por otra parte, las metas alcanzadas a nivel comunitario van a tener una repercusión enorme y directa en el devenir diario de las familias que las integran, al trasladar los valores y principios cooperativos asumidos a dicho entorno. Esto va a contribuir positivamente al bienestar de sus componentes, su consolidación como núcleo y a un cambio en los roles tradicionalmente asignados a la mujer, dirigidos hacia una mayor igualdad y respeto.

Por último, la consecución de los objetivos planteados en las perspectivas anteriores y las interrelaciones entre los mismos (las cuáles pueden consultarse en la figura $\mathrm{n}^{\circ}$. 2) posibilitarían la realización personal y profesional de la mujer indígena campesina, mejorando, entre otras cosas, su motivación, valoración y confianza en sí misma. 


\subsection{Mapa estratégico}

Figura 2. Mapa estratégico.

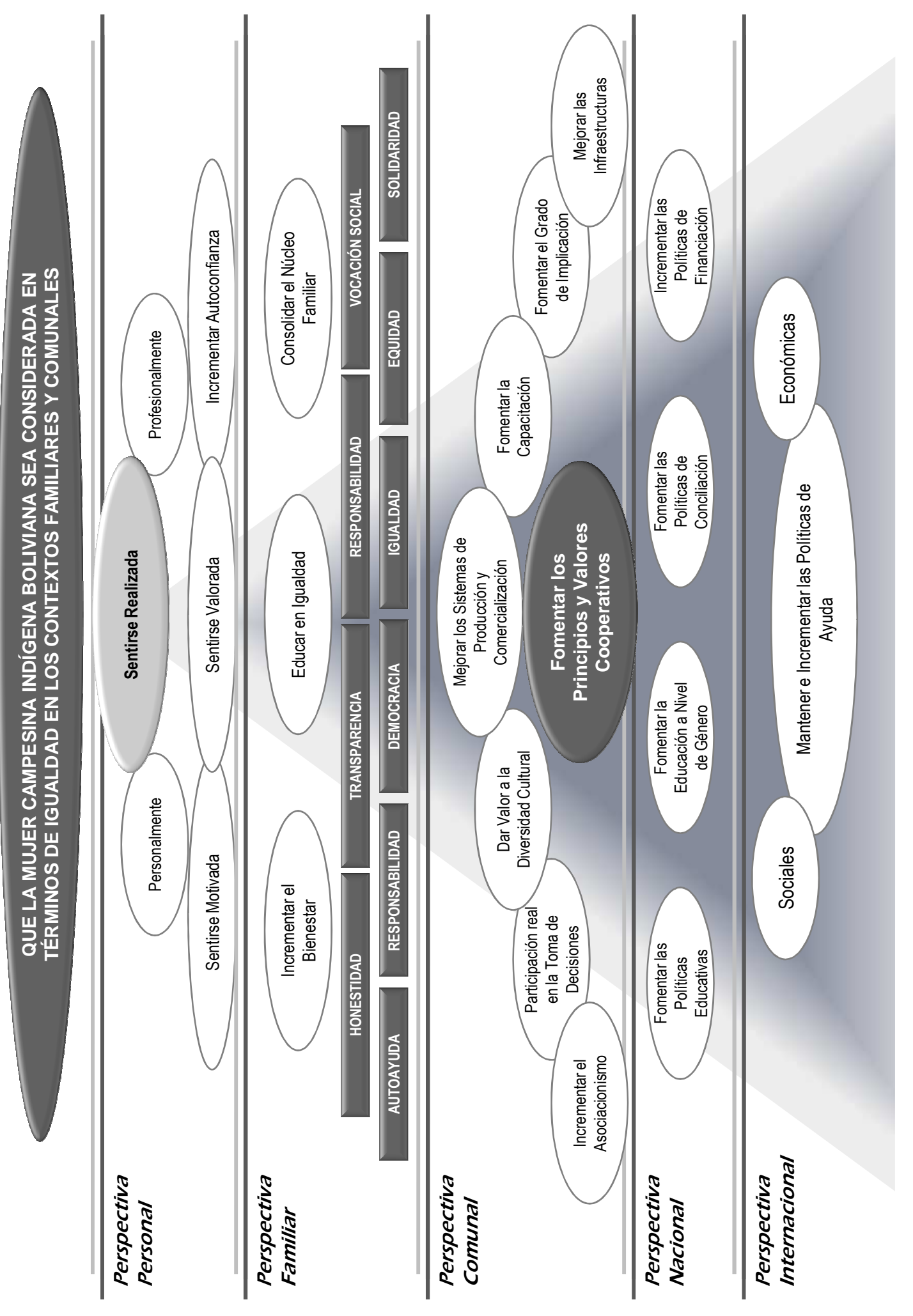

Fuente: Elaboración propia 


\section{CONCLUSIONES Y FUTURAS LÍNEAS DE INVESTIGACIÓN}

A pesar de los esfuerzos conseguidos recientemente en América Latina en materia de diversidad, los intereses y las preocupaciones de determinados grupos, entre ellos, mujeres y comunidades indígenas, no se encuentran, con carácter general, representados a los niveles de formulación de políticas, siendo la influencia de dichos colectivos aún muy limitada en lo que respecta a la toma de decisiones fundamentales en las esferas social, económica y política.

Esta realidad, se traslada al contexto de la mujer indígena campesina en Bolivia, que encara grandes dificultades en su vida cotidiana, en el interior de sus familias y comunidades, impidiendo su pleno desarrollo y obstaculizando la complementariedad de su labor personal y productiva.

Ante un contexto de tales circunstancias, en el que queda patente el papel secundario ejercido por la mujer indígena campesina boliviana, se hace necesaria la definición y puesta en marcha de una estrategia que conjugue los diferentes ámbitos de la situación analizada y que genere sinergias para la creación de valor.

En este sentido, se ha planteado el mapa estratégico como una herramienta, cuya arquitectura de causa y efecto vincula a las cinco perspectivas de actuación propuestas (personal, familiar, comunal, nacional e internacional), y en las que de manera transversal, están presentes los principios y valores cooperativos, contribuyendo a la creación de valor y a la consecución de los objetivos y de la visión planteada: "Que la mujer indígena campesina boliviana sea considerada en términos de igualdad en los contextos familiares y comunales", en definitiva, una mujer segura de sí misma, respetada por sus semejantes, capacitada, con voz y voto en la toma de decisiones y que consiga compatibilizar realmente su vida profesional y personal.

De esta forma, consideramos la elaboración y concreción del mapa estratégico como una de las principales conclusiones del presente artículo. No obstante, el alcance de los objetivos propuestos, conducentes al logro de la visión planteada, debe llevarse a cabo mediante la implementación de iniciativas concretas. Por ello, proponemos la creación de una asociación que promueva el objetivo de igualdad de las mujeres, gestionando dichas actuaciones e involucrando a los posibles participantes implicados en este reto (universidad, 
organismos públicos, comunidades,...). Dicha asociación basaría su funcionamiento en ideas y valores propios del cooperativismo ya que, como se ha argumentado anteriormente, la filosofía cooperativa permite resolver problemas de grupos sociales en desventaja mediante la puesta en común de esfuerzo, sacrificio y de una especial dosis de solidaridad. Solidaridad que, bien dirigida, permitiría el desarrollo y progreso de la sociedad en general y, en este caso, de la mujer indígena campesina en Bolivia.

Para terminar, comentar que, aunque el mapa estratégico supone el punto de arranque esencial para la definición de la estrategia, somos conscientes de que el alcance de su total eficacia implica la elaboración de un cuadro de mando integral, el cual, junto con la aplicación de esta misma metodología a otros ámbitos y colectivos, constituirán nuestras principales líneas de investigación a corto y medio plazo.

\section{BIBLIOGRAFÍA}

ACI (Alianza Cooperativa Internacional). En: http:// www.ica.coop.org

AECID (Agencia Española de Cooperación Internacional para el Desarrollo). En: www.aecid.es

BAREA, J y MONZON, J. L. Libro Blanco de la economía social en España. Madrid: Ministerio de Trabajo y Seguridad Social, 1992

BEL, P., BUENDIA, I. y RODRIGO, M. Las cooperativas y su relación con los fondos estructurales de la Unión Europea: un estudio de la política de desarrollo rural”, CIRIECEspaña, Revista de Economía Pública, Social y Cooperativa, no 35, 2000, pp. 103-126

BOJANIC, A. y OTROS. Demandas campesinas. Manual para el análisis participativo. La Paz: Embajada Real de los Países Bajos, 1994

BUENDIA, I. (1999): La Integración Comercial de las Sociedades Cooperativas. Madrid: CES (Consejo Económico Social), 1999

CABALEIRO, M. J. y FERNANDEZ-FEIJÓO, B. Sustentabilidad y cooperativismo: una propuesta metodológica basada en un proyecto real. Revista de Estudios Cooperativos (REVESCO), nº 92, 2007, pp. 72-106

CARRASCO, M. La nueva estructura de Fondos Propios para las cooperativas agroalimentarias. Huelva: Editorial Dirección de Trabajo Asociado y Empleo de Junta Andalucía, 1993 
CIRUELA. A. M. Diversificación de la actividad agraria hacia el turismo rural. Un modelo de decisión basado en sociedades cooperativas oleícolas. CIRIEC-España, Revista de Economía Pública, Social y Cooperativa, nº 61, 2008, pp. 205-232

CIRUELA, A. M. y OTROS. La mujer campesina-indígena. Málaga: Hipocampo Ediciones, 2011

COMUNIDAD EUROPEA. Resolución sobre la contribución de las cooperativas al desarrollo regional. Diario Oficial de las Comunidades Europeas (DOCE), n. C 246, $14 / 09 / 1987$

COMUNIDA EUROPEA. Resolución sobre la contribución de las cooperativas al desarrollo regional. Diario Oficial de las Comunidades Europeas (DOCE), n. C 61, 11/02/1994

CONFERENCIA EUROPEA DE SALAMANCA (2002). En:

http://ec.europa.eu/enterprise/entrepreneurship/coop/conferences/doc/salamanca-declaracionfinale-es.pdf

COQUE, J. Industrialización en el entorno local a través de sociedades cooperativas. Revista de Estudios Cooperativos (REVESCO), nº 68, 1999, pp. 119-137

DIARIO EL DIA. Bolivia. En: http://www.eldia.com.bo/index.php?cat=357\&pla=3\&id_articulo=55668

DÍAZ, M. y OTROS. Diagnóstico de la gobernabilidad, género y participación política de las mujeres: Región Andina. Santo Domingo: Instituto Internacional de Investigaciones y Capacitación de las Naciones Unidas para la Promoción de la Mujer, 2006

GALLARDO, E. Liderazgo político de las mujeres en el ámbito local. Santo Domingo: Instituto Internacional de Investigaciones y Capacitación de las Naciones Unidas para la Promoción de la Mujer, 2006

GARCÍA, E. Los Principios Cooperativos en el seno de la almazara cooperativa andaluza. Revista de Estudios Cooperativos (REVESCO), nº 70, 2000, pp.103-123

GARCIA-GUTIÉRREZ, C. Cooperativismo y desarrollo local. Revista de Estudios Cooperativos (REVESCO), nº 68, 1999, pp. 33-46

INE. Instituto Nacional de Estadística de Bolivia. En: http://www.ine.gov.bo, 2002

JULIÁ, J.F. y MARÍ, S. Agricultura y desarrollo rural. Contribuciones de las cooperativas agrarias. CIRIEC-España, Revista de Economía Pública, Social y Cooperativa, no 41, 2002, pp. $25-52$

JULIA, J.F., GARCIA, G. y MELIÁ, E. Internet, cooperativismo agrario y desarrollo rural. El caso de las cooperativas de la Comunidad Valenciana. CIRIEC-España, Revista de Economía Pública, Social y Cooperativa, no 55, 2006, pp. 221-251 
KAPLAN, R. S. y NORTON, D.P. The balanced scorecard - Measures that drive performance. Harvard Business Review 70 (1), 1992 pp. 71-79

KAPLAN, R. S. y NORTON, D.P. Putting the balanced scorecard to work. Harvard Business Review, 71(5), 1993, pp.134-142

KAPLAN, R. S. y NORTON, D.P. Linking the balanced scorecard to strategy. California Management Review, 39(1), 1996a, pp. 53-79

KAPLAN, R. S. y NORTON, D.P. Using the balanced scorecard as a strategic management system. Harvard Business Review, 74(1), 1996b, pp. 75-85

MÄCKELMANN, M. Descentralización y Reforma Constitucional en la Región Andina. Principales conclusiones del Seminario Internacional, Ecuador: FLACSO, 2007

MARTINSONS, M., DAVISON, R., y TSE, D. The balanced scorecard: A foundation for the strategic management of information systems. Decision Support Systems, 25(1), 1999, pp. $71-88$

M.A.S (Movimiento al Socialismo). Programa de Gobierno. En: http://www.archivochile.com/Portada/bol_elecciones05/bolelecciones0009.pdf

MINISTERIO DE EDUCACION BOLIVIA. En: http://www.minedu.gob.bo/

MONTENEGRO, S. El Estado, las mujeres y la lucha para la ciudadanía en América Latina. Notas para entender la exclusión y la desigualdad de género. En: http://www.movimientoautonomodemujeres.org/downloads/20.pdf, 2008

MOZAS, A. y RODRIGUEZ, J.C. La economía social: agente del cambio estructural en el ámbito rural. Revista de Desarrollo Rural y Cooperativismo Agrario, n 4, 2000, pp. 7-18

MOZAS, A. y BERNAL, E. Desarrollo territorial y Economía social. CIRIEC-España, Revista de Economía Pública, Social y Cooperativa, nº 55, 2006, pp.125-140

INSTITUTO NACIONAL DE ESTADÍSTICA DE BOLIVIA (INE). En: http://www.ine.gov.bo/default.aspx

REAL ACADEMIA DE LA LENGUA (RAE). Diccionario de la lengua española. Vigésima segunda edición. En: www.rae.es

RODRIGUEZ, J.C. Desarrollo local por Cooperativas: Diversificación productiva en el ámbito rural andaluz. El caso de Bedmar. Revista de Estudios Cooperativos (REVESCO), $n^{\circ} 68,2002, p p .173-190$

SANCHIS, J.R. Análisis Estratégico de la Empresa Cooperativa. Valencia: Editorial Tirant Lo Blanch, 1995 
TOUS, D. La cultura organizacional como factor de éxito en las empresas cooperativas", Actas 1er Congreso sobre Cooperativismo Español, Edita Fundación Fernando Garrido Tortosa. Osuna, 1999, pp. 571-587

UDAPE (Unidad de análisis de políticas sociales y económicas). Bolivia. En: http://www.udape.gob.bo/ 